\title{
Depth-Sensitive Raman Investigation of Metal-Oxide-Semiconductor Structures: Absorption as a Tool for Variation of Exciting Light Penetration Depth
}

\author{
Paweł Borowicz ${ }^{1,2}$ \\ ${ }^{1}$ Institute of Electron Technology, Aleja Lotników 36/42, 02-668 Warsaw, Poland \\ ${ }^{2}$ Institute of Physical Chemistry, Polish Academy of Sciences, Kasprzaka 44/52, 01-224 Warsaw, Poland \\ Correspondence should be addressed to Paweł Borowicz; psborowicz@wp.pl
}

Received 27 September 2015; Accepted 10 November 2015

Academic Editor: Christoph Krafft

Copyright (C) 2016 Paweł Borowicz. This is an open access article distributed under the Creative Commons Attribution License, which permits unrestricted use, distribution, and reproduction in any medium, provided the original work is properly cited.

\begin{abstract}
Presented work focuses the attention on two regions of MOS structure placed in the vicinity of the semiconductor/dielectric interface, in particular: on part of dielectric layer and thin layer of the substrate. In the presented work the application of absorption as a tool that can vary the absorption depth of excitation light into the semiconductor substrate is discussed. The changes of the absorption depth of visible light allows to obtain Raman signal from places in the substrate placed at different distances from the dielectric/semiconductor interface. The series of Raman spectra obtained from visible excitation in the case of varying absorption depth allowed to analyze the structure of the substrate as a function of distance from the interface. Deep ultraviolet Raman study regarding part of silicon dioxide layer placed directly at the interface is not discussed so far which makes the analysis of the structure of this part of dielectric layer possible. Comparison of reported in this work Raman data with structure of silicon/silicon dioxide interface obtained from other experimental techniques proves the applicability of proposed methodology.
\end{abstract}

\section{Introduction}

The progress in miniaturization of Metal-oxide-semiconductor- (MOS-) type electronic devices results in limitation of active area of semiconductor substrate to the thin layer placed in the vicinity of interface between semiconductor substrate and dielectric layer. An example of such device is High Electron Mobility Transistors (HEMTs). The thickness of active area in this device is limited to several dozen nanometers [1].

Raman spectroscopy detects small shifts in frequencies of normal modes caused by small differences between parameters of crystal or molecular structure like bond lengths or bond angles. This accuracy makes from this experimental technique a very efficient tool for structural study. An example of this type of application of Raman spectroscopy is delivered by study of changes in semiconductor structure caused by implantation [2].

Optical microscopy is the experimental technique which offers high spatial resolution. The transverse resolution is determined by diffraction limit of microscopic objective. The diameter of Airy spot can be calculated according to Rayleigh or Sparrow criteria [3]. In the case of the microscopic lenses with high numerical aperture $\left(N_{A}\right), N_{A}=0.55$, the dimension of Airy spot is placed in the range between $500 \mathrm{~nm}$ and $700 \mathrm{~nm}$ [3]. Spatial resolution of Raman microscopy was applied in the investigation of spatial distribution of such parameters like mechanical stress in semiconductor substrate $[4,5]$ or channel temperate in HEMTs $[6,7]$. The thermal effect observed in HEMTs is caused by self-heating present in the case of current flow between source and drain of the transistor.

The axial dimension of the focus depends also on $N_{A}$. The distribution of the intensity across the laser beam can be described by Gaussian function. The axial dimension of the laser beam focus in the case of confocal microscopes is not smaller than $1 \mu \mathrm{m}$ [8]. The thickness of active area in today's electronic devices is at the least order of magnitude smaller than axial dimension of laser beam focus determined from geometrical optics. Because of this it is necessary to 
introduce the procedure that can avoid the limitation coming from geometrical optics.

The most important property that can help to overcome the limitation of axial diameter of laser beam focus is absorption. Absorption coefficient of each material is a function of wavelength of incident light. It means that one can change the penetration depth of the light into the material by choice of the irradiation wavelength. The dependence between wavelength and absorption of the material was applied for investigation of ohmic contacts with additional carbon layer formed at different temperatures [9]. Silicide film mixed with carbon atoms is transparent for visible light, because this visible irradiation of the ohmic contact through silicide layer causes the Raman scattering in carbon layer placed between silicide film and silicon carbide substrate. The deep-ultraviolet excitation applied in the same configuration cannot reach the above-mentioned carbon layer due to strong absorption of the silicide layer mixed with carbon structures. Therefore Raman scattering excited in deep-ultraviolet spectral range delivers information about two types of carbon species:

(i) carbon layer which is built on the free surface of silicides due to carbon atom diffusion;

(ii) carbon clusters placed inside of silicide layer [9].

The other problem which was investigated by means of extraction of signal generated in thin layer from large background is related to properties of the interface between silicon carbide $(\mathrm{SiC})$ and dielectric layer. The Near Interface Traps (NITs) in the MOS-type structures can decrease the mobility of charge carriers even by two orders of magnitude. Extensive study of the properties of $\mathrm{SiC} / \mathrm{SiO}_{2}$ interface showed that carbon plays very important role in formation of the defects that can be candidates for NITs [10-13]. The most important Raman bands generated by species built from carbon atoms [14] are placed in the same range of Raman shift as two-phonon spectrum of different polytypes of $\mathrm{SiC}$ $[15,16]$. Application of two different excitation wavelengths, in particular visible and ultraviolet, made the extraction of the scattering coming from the interface from background which is formed by two-phonon Raman scattering in SiC substrate possible. The extraction was possible due to significantly different penetration depths of exciting radiation from both used spectral ranges [17].

The other area where the decreasing of two-phonon Raman scattering plays a key role is related to Raman study of properties of dielectric layer. The problem was discussed in the literature for the system composed of $\mathrm{Si}$ substrate and $\mathrm{SiO}_{2}$ layer. Standard configuration of Raman apparatus includes excitation in visible spectral range. In this case significant two-phonon signal generated in $\mathrm{Si}$ substrate is observed [18]. The intensity of this second-order Raman scattering is strong enough even to mask the signal form $\mathrm{SiO}_{2}$ layer [19]. Application of deep-ultraviolet excitation makes possible the observation of Raman scattering generated in $\mathrm{SiO}_{2}$ layer [20]. The increase of $\mathrm{Si}$ absorption due to change of the excitation wavelength from visible spectral range to deep-ultraviolet results in reduction of radiation penetration depth by about 30 times. In turn, the intensity of twophonon Raman scattering coming from Si substrate becomes negligible and the signal from silicon oxide layer becomes detectable. This was shown for $\sim 50 \mathrm{~nm}$ thick $\mathrm{SiO}_{2}$ layer placed on Si substrate by comparison with bulk material which was commercially available quartz glass Suprasil I [20]. The price to pay for this advantage is long irradiation time. The reason for this "price" is small efficiency of Raman effect in the case of $\mathrm{SiO}_{2}$ combined with small thickness of investigated material. The typical thickness of $\mathrm{SiO}_{2}$ layer of today's electronic structures is about two orders of magnitude smaller than the axial dimension of the focus of laser beam.

This work focuses the attention on the properties of thin layer of semiconductor substrate in the vicinity of semiconductor/dielectric interface. The $\mathrm{Si} / \mathrm{SiO}_{2}$ system is used as an example. The change of the power density of exciting light on the sample results in change of effective absorption depth. Effective absorption depth is the thickness of the investigated material which is active from the point of view of measured Raman signal under certain power density. Since the definition of the effective absorption depth is a crucial point in the interpretation of experimental data it will be discussed in detail in the next chapter Experimental where also the methodology of data analysis is described. The systematic change of power density makes possible to record Raman signal from material with different thickness. As a result one can get depth profile of structural properties of investigated material.

Another point that will be discussed here is the appearance of crystal-like structures of silicon dioxide that should be placed at the $\mathrm{Si} / \mathrm{SiO}_{2}$ interface [21]. As was mentioned above application of deep-ultraviolet excitation in order to reduce two-phonon signal from $\mathrm{Si}$ substrate was discussed for amorphous part of $\mathrm{SiO}_{2}$ layer [20]. However, Raman signal observed for this type of excitation contains also traces of narrow lines. These traces will be compared here with Raman spectra reported for crystalline forms of silicon dioxide.

The paper is organized according to the following outline. Section 2 presents method of sample preparation and their characterization, Raman apparatus, and methodologies of data analysis and measurements. The special attention was paid to two aspects:

(i) the description of the mathematical model which links the power density of irradiation with thickness of the layer of material from which the Raman scattering is recorded;

(ii) the discussion of two experimental parameters which are changed if the power density of exciting light is varied: half-angle of the maximum cone of light collected by microscope objective and the dimension of the laser-beam spot.

Section 3 presents the measured data. The results of the investigation are discussed in Section 4. 


\section{Experimental}

2.1. Samples Preparation and Characterization. Silicon dioxide films were manufactured in Division of Silicon Microsystem and Nanostructure Technology (Institute of Electron Technology, Warsaw, Poland). Three-inch diameter p-type silicon wafers were used as substrates. Samples were characterized by means of spectroscopic ellipsometry, transmission electron microscopy, and transmission/reflection spectroscopy. Details of sample preparation and characterization were already presented in the literature [20].

2.2. Raman Apparatus. Raman spectra were measured with micro-Raman spectrometer MonoVista 2750i (Spectroscopy \& Imaging $\mathrm{GmbH}$, Germany).

Microscopy part of the spectrometer is based on fluorescence microscope type BX-51 (Olympus, Japan). The microscope is equipped with four objectives:

(i) three of them (magnification: 100x, 50x and 20x) are working in visible (VIS) spectral range;

(ii) one objective (magnification 40x) is designed for deep-ultraviolet (deep-UV) spectral range.

Images from microscope are recorded with imaging camera TM 2040 GE (JAI, Japan). Motorized stage (Ludl Electronic, USA) makes the following types of spatially resolved measurements possible:

(i) line scanning along each coordinate: $x, y$, and $z$;

(ii) two-dimensional mapping: $x y, y z$, and $x z$;

(iii) three-dimensional mapping $x y z$.

Spectroscopy part of the setup is based on imaging spectrograph SpectraPro 2750i equipped with liquid nitrogen cooled spectroscopy CCD camera LN/2048 × 512B/IUVAR, Spec-10 System (Princeton Instruments USA). The camera has maximum efficiency in UV spectral range. The spectrograph has three diffraction gratings:

(i) two of them (1800 grooves/mm and 2400 grooves/ $\mathrm{mm}$ ) are blazed in visible spectral range;

(ii) one grating (3600 grooves $/ \mathrm{mm}$ ) is blazed in ultraviolet.

Large focal length of the spectrograph $(750 \mathrm{~mm})$ allows high spectral resolution combined with single pass of the radiation through the spectrograph. The spectral resolution of the apparatus is equal to about $0.1 \mathrm{~cm}^{-1}$ for VIS spectral range and about $1 \mathrm{~cm}^{-1}$ in deep-UV.

In the case of visible excitation the combination of objective with magnification equal to $100 \mathrm{x}$ and grating with 2400 grooves $/ \mathrm{mm}$ was used. The combination of deep-UV objective and grating with 3600 grooves/mm was applied to record the spectra excited in deep-UV.

As excitation sources two continuous work (CW) lasers were used. Raman scattering in VIS spectral range was excited with $\mathrm{Ar}^{+}$laser INNOVA 90C FRED (Coherent Inc., USA). In particular the line $488 \mathrm{~nm}$ was used. Deep-UV excitation was done by means of semiconductor laser FQCW-266-10 (CryLas $\mathrm{GmbH}$, Germany). The wavelength of laser line was equal to $266 \mathrm{~nm}$.

The power of the excitation light was not larger than $1 \mathrm{~mW}$ on the sample for each excitation wavelength. The position of one-phonon Si line depends on the temperature; in particular it is shifted by about $2 \mathrm{~cm}^{-1}$ towards smaller values of Raman shift if the temperature increases from room temperature to $100^{\circ} \mathrm{C}[22]$. The power of exciting light should be set within the range which makes it possible to avoid local heating of the sample caused by absorption of the exciting light. Applied in this work power of exciting light is adjusted within the range used for investigation of the stress in semiconductors. For example, the power of the exciting light on the sample used for stress mapping in porous Si microcapsules was equal to $1 \mathrm{~mW}$ [23]. The power of laser line on the sample used in investigation of self-heating effect in electronic devices is equal even to $5 \mathrm{~mW}[7,24,25]$.

2.3. Model. As was mentioned in Introduction variation of the power density results in changes of thickness of the material from which the scattering is collected. The type of measurement called line scan $z$ allows to record the series of Raman spectra for different power density of irradiation light on $\mathrm{Si} / \mathrm{SiO}_{2}$ interface. This set of different values of power density is reflected in variation of thickness of material from which Raman signal is collected. This thickness will be called effective absorption depth, $\delta_{\text {eff }}$.

The concept of tuning of the thickness of material from which Raman signal is collected is based on the fluorescence measurement in reflection. This concept was applied in Multifunctional Spectrofluorimetric System designed by Jasny ("focusing and collimating system type A") [26]. In this configuration angle between optical axes of exciting and analyzing setup was equal to $30^{\circ}$. It means that the luminescence was measured in the configuration similar to the back scattering. Measurements were performed in solutions. The thickness of the layer where the excitation light was effectively absorbed was determined by concentration of absorbing agent, because the intensity of exciting light was constant. In the case of high concentration of absorbing species the thickness absorbing layer was tended to monomolecular film.

In the case of Raman scattering in solid material (i.e., $\mathrm{Si}$ ) excited with laser line the absorption is determined by the wavelength of exciting light and it is constant for each wavelength. In order to tune the effective absorption depth the power density of the irradiation light must be varied. Tuning of the power density of exciting light in the case of constant absorption results in the same effect as tuning of the absorption for constant power density. In particular the thickness of the material where the incoming light is effectively absorbed is changed. Important is the question about the lower limit of the thickness of the film where all photons from incoming light should be absorbed. This layer should have the same properties as whole material. In the case of crystalline media the smallest part of material which has the same properties as large crystals is defined by unit cell. It means that in the case of small density of irradiation light 
the thickness of absorbing layer should be compared with dimension of unit cell of the investigated material. In the case of crystalline silicon this dimension is equal to about $0.5 \mathrm{~nm}$.

The crucial problem in quantitative data analysis is the development of the model which allows to calculate values of effective absorption depth resulting from changes of power density of irradiation light on the sample. The absorption of exciting and scattered light must be taken into account. The model which links the variation of power density of irradiation light with effective absorption depth is presented below.

Let us start with short overview of basic knowledge necessary for the development of the announced above mathematical model. The absorption of material is described by Lambert law [27]:

$$
I(l)=I_{0} e^{-\alpha l}
$$

where $I_{0}$ denotes the intensity of incident light, $l$ is the optical pathway through the material, $I(l)$ is the intensity of the light after the pathway equal to $l$, and $\alpha$ is the absorption coefficient of the material. In many applications the base equal to 10 is used instead of exponential function. In such a case the Lambert law has the form

$$
I(l)=I_{0} 10^{-B}=I_{0} 10^{-\beta l},
$$

where quantity $B$ is called absorbance and $\beta$ denotes an extinction coefficient. Lambert law can be expressed in terms of imaginary part of complex refractive index. The complex index of refraction $n_{c}$ has the form

$$
n_{c}=n-i k,
$$

where $n$ and $k$ denote real and imaginary parts of complex refractive index, respectively. Both $n$ and $k$ are functions of wavelength. The sign “-” in (3) results in positive value of damping coefficient of electric field $k$ for materials which do not amplify the light. Since the intensity is proportional to the square of electric field amplitude absorption coefficient $\alpha$ can be expressed as a function of damping coefficient $k$ by the following equation:

$$
\alpha=\frac{2 \omega k}{c},
$$

where $\omega$ denotes the circular frequency and $c$ is the velocity of light. Taking into account dependencies between circular frequency $\omega$, frequency $\nu$, and wavelength $\lambda$ one can present (4) in the following form:

$$
\alpha=\frac{2(2 \pi \nu) k}{c}=\frac{4 \pi c k}{\lambda c}=\frac{4 \pi k}{\lambda} .
$$

Penetration depth is defined as thickness of absorbing material which corresponds to the decrease of the quantity describing electromagnetic wave by $e$ times [28]. Since intensity is important for further analysis the attention will be focused on parameters related to intensity of the light. In such a case the penetration depth $\delta$ is given by following equation:

$$
\delta=\frac{\lambda}{4 \pi k(\lambda)}=\alpha^{-1}
$$

The imaginary part of refractive index used in (6) has the form $k(\lambda)$ in order to emphasize the fact that the damping coefficient is a function of wavelength. Taking into account values of Si damping coefficient for green lines of $\mathrm{Ar}^{+}$laser (wavelengths of laser lines usually used in Raman experiments: $488 \mathrm{~nm}$ and $514 \mathrm{~nm}$ ) [29] one obtains penetration depth equal to about $0.7 \mu \mathrm{m}$. The application of deep-UV excitation (typical wavelengths of laser lines: $244 \mathrm{~nm}$ or $266 \mathrm{~nm}$ ) reduces the value of $\delta$ to several dozen nanometers [29].

Now we can move to the definition of effective absorption depth. This quantity is important in the case of micro-Raman study of semiconductor substrates because it determines the thickness of material which is investigated. Effective absorption depth can be calculated from properties of spectral CCD camera used to record the Raman signal and the damping coefficient $k$. The following approximation will be used in calculation of effective absorption depth: due to small difference between wavelengths of exciting and scattered light, the value of damping coefficient $k$ will be taken equally for excitation and scattering.

Now we focus the attention on crystalline silicon as an example of absorbing material. The first step to determine the effective absorption depth is to show that the thickness of $\mathrm{Si}$ from which the Raman scattering is collected depends on the number of incident photons. One can express the number of photons reaching the depth equal to $z$ by the following equation:

$$
n(z)=n_{0} e^{-\alpha z}
$$

where $n_{0}$ is the number of incident photons for unit area. Value $z=0$ corresponds to the interface between silicon substrate and silicon dioxide layer. The maximal depth corresponds to the thickness of material which is passed only by one photon: $z=z_{\max } \rightarrow n(z)=1$. It means that one can change $z_{\max }$ by varying the number of incident photons $n_{0}$. Equation (7) can be rewritten as a condition for $z_{\max }$ :

$$
z_{\max }=\frac{\ln \left(n_{0}\right)}{\alpha} .
$$

The condition specified by $\left(7^{\prime}\right)$ shows that one can change the thickness of the material which gives the contribution to Raman scattering by varying the number of incident photons per unit area $n_{0}$.

Let us move now to the calculation of effective absorption depth in the case of Raman scattering. CCD cameras used in spectroscopic measurements have 16-bit analog-digital conversion. It means the maximum intensity of the Raman line that can be accumulated is equal to $2^{16}=65536$ counts. Further extension of accumulation time results in saturation of line intensity. It means that the maximum cannot be obtained because the upper part of the line (over 65536 counts) is cutoff. The intensity of scattered light $I(l)$ as a function of thickness of material $l$ is given by following equation:

$$
I(l)=I_{0} \varphi_{0} \int_{0}^{l} e^{-2 \alpha z} d z=I_{0} \varphi_{0} \frac{1-e^{-2 \alpha l}}{2 \alpha} .
$$


In (8) $I_{0}$ denotes the intensity of excitation and $\varphi_{0}$ describes the efficiency of the observed Raman effect. Integration from 0 to $l$ gives the intensity of Raman scattering measured from the layer whose thickness is equal to $l$. The expression $\exp (-2 \alpha z)$ under the integral described the statistical weight of the contribution of the scattering coming from the material placed at the depth equal to $z$. Factor 2 in the argument of exponential function in (8) was introduced because scattered light is absorbed in the same manner as exciting light. At this point the approximation concerning the equality of damping coefficient for exciting and scattered light is introduced to the model. The intensity reaches its maximal value equal to 65536 when the thickness is equal to $\delta_{\mathrm{eff}}^{\max }$. Equation (8) takes in this case the following form:

$$
\begin{aligned}
I\left(\delta_{\mathrm{eff}}^{\mathrm{max}}\right) & =I_{0} \varphi_{0} \int_{0}^{\delta_{\mathrm{eff}}^{\max }} e^{-2 \alpha z} d z=I_{0} \varphi_{0} \frac{1-e^{-2 \alpha \delta_{\mathrm{eff}}^{\max }}}{2 \alpha} \\
& =65536 .
\end{aligned}
$$

In practice it is convenient to explain $\delta_{\text {eff }}^{\max }$ by multiple of value of penetration depth $\delta$ defined by (6). The value of $I(n \delta)$ efficiently tends to maximal value of intensity with increase of $n$. In particular the values of $I(n \delta) / I\left(\delta_{\mathrm{eff}}^{\max }\right)$ ratio are equal: 0.865 for $n=1,0.982$ for $n=2$, and 0.998 for $n=3$. The standard deviation of the intensity of Raman line can be calculated from Poisson distribution, because photon statistics is based on this distribution. The standard deviation is equal to the square root of the intensity understood as a number of counts obtained for Raman shift equal to maximum position of the line. In the case of maximal value of intensity measurable by 16-bit CCD camera the standard deviation is equal to $\Delta\left(I\left(\delta_{\mathrm{eff}}^{\max }\right)\right)=256=(65536)^{1 / 2}$. This standard deviation is equal to about $0.4 \%$ of the maximum measurable intensity. The ratio $\left(I\left(\delta_{\mathrm{eff}}^{\max }\right)-\Delta\left(I\left(\delta_{\mathrm{eff}}^{\max }\right)\right)\right) / I\left(\delta_{\mathrm{eff}}^{\max }\right)$ is equal to 0.996 . It means that $I(n \delta)$ for $n=3$ is placed within the range $I\left(\delta_{\mathrm{eff}}^{\max }\right) \pm \Delta\left(I\left(\delta_{\mathrm{eff}}^{\max }\right)\right)$.

To sum up, assuming $\delta_{\text {eff }}^{\max }=3 \delta$, one obtained the intensity of Raman scattering which differs from $I\left(\delta_{\text {eff }}^{\max }\right)=$ 65536 by value smaller than standard deviation $\Delta\left(I\left(\delta_{\mathrm{eff}}^{\max }\right)\right)$.

In the case of crystalline Si for excitation wavelength equal to $488 \mathrm{~nm}$ the maximum of effective absorption depth is equal to $2.2 \mu \mathrm{m}$. The change to deep-UV excitation (wavelength $244 \mathrm{~nm}$ or $266 \mathrm{~nm}$ ) reduces $\delta_{\text {eff }}^{\max }$ for crystalline Si to the value from the range between $100 \mathrm{~nm}$ and $200 \mathrm{~nm}$.

The next problem is how to link the effective absorption depth $\delta_{\text {eff }}$ with intensity of Raman line if the intensity does not reach value $I\left(\delta_{\text {eff }}^{\max }\right)=65536$. Replacing $l$ by $\delta_{\text {eff }}$ in (8) one obtains the following expression:

$$
I(l)=I_{0} \varphi_{0} \int_{0}^{\delta_{\mathrm{eff}}} e^{-2 \alpha z} d z=I_{0} \varphi_{0} \frac{1-e^{-2 \alpha \delta_{\mathrm{eff}}}}{2 \alpha} .
$$

Dividing $\left(8^{\prime \prime}\right)$ by $\left(8^{\prime}\right)$ one obtains the intensity ratio $I\left(\delta_{\text {eff }}\right) / I\left(\delta_{\text {eff }}^{\max }\right)$ :

$$
\frac{I\left(\delta_{\mathrm{eff}}\right)}{I\left(\delta_{\mathrm{eff}}^{\max }\right)}=\frac{1-e^{-2 \alpha \delta_{\mathrm{eff}}}}{1-e^{-2 \alpha \delta_{\mathrm{eff}}^{\max }}}
$$

Expanding $\exp (-2 \alpha z)$ in power series for $z=\delta_{\text {eff }}$ and $z=$ $\delta_{\text {eff }}^{\max }$ one obtains approximate expression for intensity ratio $I\left(\delta_{\text {eff }}\right) / I\left(\delta_{\text {eff }}^{\max }\right)$ :

$$
\frac{I\left(\delta_{\mathrm{eff}}\right)}{I\left(\delta_{\mathrm{eff}}^{\max }\right)}=\frac{\delta_{\mathrm{eff}}}{\delta_{\mathrm{eff}}^{\max }}
$$

From $\left(9^{\prime}\right)$ one can obtain the following expression for $\delta_{\text {eff }}$ :

$$
\delta_{\mathrm{eff}}=\frac{I\left(\delta_{\mathrm{eff}}\right)}{I\left(\delta_{\mathrm{eff}}^{\max }\right)} \delta_{\mathrm{eff}}^{\max }=3 \frac{I\left(\delta_{\mathrm{eff}}\right)}{I\left(\delta_{\mathrm{eff}}^{\max }\right)} \delta .
$$

In (10) the obtained earlier approximation for maximum of effective absorption depth $\delta_{\mathrm{eff}}^{\max }=3 \delta$ was introduced.

2.4. Measurement Methodology. Two types of measurements were performed. In the case of deep-UV excitation the laser beam was focused on the $\mathrm{Si} / \mathrm{SiO}_{2}$ interface. Due to small intensity of the signal long time of irradiation was applied. The exposition time of single spectrum accumulation was equal to 1 hour.

The second type of measurement is called line scan $z$. This type of measurement was performed for VIS excitation $(\lambda=$ $488 \mathrm{~nm}$ ). The direction perpendicular to the surface of interface is marked with $z$ coordinate. The following sequence of steps was necessary to perform line scan along $z$ coordinate. First the laser beam was focused on the $\mathrm{Si} / \mathrm{SiO}_{2}$ interface. This position was assumed as $z=0$. Then the measurements of Raman spectra were performed for different values of $z$ coordinate. The range of coordinate which was scanned spread from $-20 \mu \mathrm{m}$ to $20 \mu \mathrm{m}$. The difference between two subsequent $z$ positions, in other words the step of the scan, was equal to $1 \mu \mathrm{m}$. The exposition time for single spectrum was equal to 1 minute.

There are two important parameters of the setup which change when the sample is moved along optical axis from the position corresponding to focal spot. These parameters are half-angle of the maximum cone of light collected by microscope objective and the power density of the laser light exciting Raman scattering. Let us now discuss how these parameters change if the sample is moved from focus position by $z=20 \mu \mathrm{m}$.

Figure 1 presents the beam path between microscope objective and the sample. The cone of the light that can be collected by objective is described by hyperboloid. The asymptote of the hyperboloid is defined by numerical aperture of the objective. The numerical aperture $N_{A}$ is the function of half-angle $\Theta$ of the maximum cone of light that can enter the microscope objective. The other important parameters of the objective are as follows:

(i) $f$ : focal length;

(ii) $w$ : working distance;

(iii) $\Phi$ : diameter of the entrance pupil;

(iv) $2 r_{0}$ : diameter of the focal spot. 


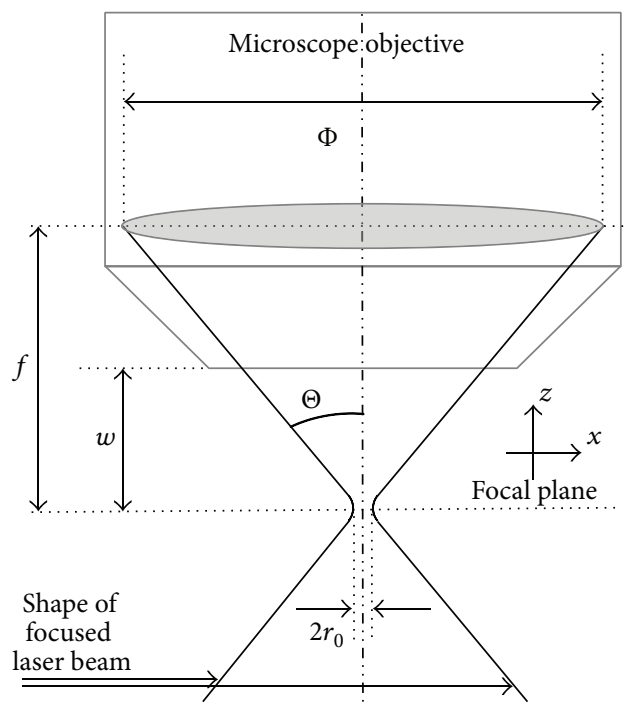

FIGURE 1: Schematic presentation of laser beam focused by microscope objective: $f$ : focal length, $w$ : working distance, $2 r_{0}$ : diameter of the focal spot, $\Theta$ : half-angle of maximum cone of light collected by microscope objective, $\Phi$ : entrance diameter of the objective, and $x, z$ : coordinates.

Numerical aperture, half-angle, focal length, and diameter of entrance pupil fulfill the following equation:

$$
N_{A}=\sin (\Theta)=\sin \left(\arctan \left(\frac{\Phi}{2 f}\right)\right) .
$$

The diameter of the focal spot can be calculated from Rayleigh or Sparrow [3] criterion for diffraction limit of Airy spot:

$$
2 r_{0}=C \frac{\lambda}{2 N_{A}},
$$

where $C=1$ in the case of Sparrow criterion or $C=1.22$ for Rayleigh criterion.

Let us start the discussion of experiment methodology from half-angle $\Theta$. In the case of Olympus objective M Plan Semi-Apochromat MPLFLN 100x used in experiment parameters $w$ and $f$ are equal to $1.0 \mathrm{~mm}$ and $1.8 \mathrm{~mm}$, respectively. Numerical aperture of this objective is equal to 0.9 . The halfangle $\Theta$ and entrance pupil diameter $\Phi$ are equal to

$$
\begin{aligned}
& \Theta=\arcsin \left(N_{A}\right)=64.16^{\circ}, \\
& \Phi=2 f \tan (\Theta)=2 f \tan \left(\arcsin \left(N_{A}\right)\right)=7.4 \mathrm{~mm} .
\end{aligned}
$$

Shift of the sample from position of focal spot towards microscope objective by distance $z$ results in change of halfangle of maximum cone of the light collected by objective. The value of half-angle $\Theta^{\prime}$ for shifted sample is equal to

$$
\Theta^{\prime}=\arctan \left(\frac{\Phi}{2(f-z)}\right) \text {. }
$$

In the case of used objective and shift equal to $z=20 \mu \mathrm{m}$ the angle $\Theta^{\prime}$ is equal to $64.31^{\circ}$. It means that half-angle changes by $0.15^{\circ}$. The value of "numerical aperture" $N_{A}^{\prime}$ in the case of being defocused by $20 \mu \mathrm{m}$ position of the sample is equal to

$$
N_{A}^{\prime}=\sin \left(\Theta^{\prime}\right)=0.90114 \text {. }
$$

The relative change of numerical aperture caused by defocusing by $20 \mu \mathrm{m}$ is equal to

$$
\sigma=\frac{\left|N_{A}^{\prime}-N_{A}\right|}{N_{A}}=0.0013 .
$$

The relative change of numerical aperture $\sigma=0.13 \%$ is smaller than the standard deviation-to-signal ratio in the case of maximum signal that can be measured with CCD camera $(0.4 \%)$.

Let us now focus the attention on the changes of spot dimension caused by defocusing. The diameter of focal spot $2 r_{0}$ calculated from Rayleigh criterion for the MPLFLN 100x objective and laser line $488 \mathrm{~nm}$ is equal to $0.331 \mu \mathrm{m}$. As reported in the literature values of focal spot should not exceed $0.7 \mu \mathrm{m}$ [3]. As was mentioned above the shape of longitudinal section of focused laser beam is described by hyperbola:

$$
\frac{x^{2}}{a^{2}}-\frac{z^{2}}{b^{2}}=1 .
$$

Let us take into account the most unfavorable option; it means $a=r_{0} \approx 0.35 \mu \mathrm{m}$. Parameter $b$ can be calculated from the slope of the hyperbola asymptote. This slope is defined by half-angle of maximum cone of the light detected by objective, in other words by numerical aperture $N_{A}$. In the case of assumed diameter of focal spot the value of $b$ parameter is equal to

$$
b=r_{0} \tan \left(90^{\circ}-\Theta\right) \approx 0.169 \mu \mathrm{m} .
$$

Since power density changes in the same way as the area of the spot the ratio $x^{2} / r_{0}^{2}$ determines changes of power density as a function of shift from position of focal spot $z$ (for focal $\operatorname{spot} z=0$ ),

$$
\frac{x^{2}}{r_{0}^{2}}=1+\frac{z^{2}}{b^{2}} .
$$

In the case of defocusing by $z=20 \mu \mathrm{m}$ the power density of exciting light decreases $\left(x^{2} / r_{0}^{2}\right)=14005$ times, which means by five orders of magnitude.

To sum up, the shift of the sample along the optical axis of microscope objective from focal plane by $20 \mu \mathrm{m}$ results in

(i) negligible change of half-angle of maximum cone of the light collected by objective;

(ii) decrease of the power density of exciting light by five orders of magnitude.

It should be also emphasized that assumption (in the calculation of power density diameter of focal spot) introduces the condition which is significantly unfavorable in comparison with Rayleigh of Sparrow criteria. 
It should be also mentioned that standard technique used to decrease the intensity of $\mathrm{cw}$ laser light does not offer this range of intensity tuning as defocusing. Standard method used to decrease $\mathrm{cw}$ laser line intensity is based on high polarization degree of this light. Introducing linear polarizer into the laser beam makes it possible to vary the intensity via changing the angle $\psi$ between direction of polarizer axis and the direction of the laser beam polarization. The changes of intensity are in this case described by $\cos ^{2}(\psi)$ (Malus' law). The change of angle $\psi$ between $0^{\circ}$ and $89^{\circ}$ corresponds to the decrease of the light intensity by 3283 times, by three orders of magnitude. This value is significantly smaller than the decrease obtained from defocusing of laser beam. Moreover in the case of polarizer application some values of $\delta_{\text {eff }}$ placed near the interface are not available for experimental study. These values of $\delta_{\text {eff }}$ are extremely interesting from the point of view of manufacturing technology of today's electronic devices.

2.5. Data Analysis. Data obtained from excitation in different spectral ranges were focused on different type of information. The spectra recorded for visible excitation were focused on one-phonon Si line. In the case of deep-UV excitation the attention was focused on Raman scattering generated in $\mathrm{SiO}_{2}$ layer. Because of this different procedures were applied to analyze the data recorded for excitation in different spectral ranges.

In order to obtain the parameters like maximum position and FWHM of one-phonon Si line the following mathematical procedure was applied. In the first step of the procedure all signals except silicon line were treated as background. This background was removed with spline polynomials. After background removal Lorentzian profile was fitted to the one-phonon Si line. Maximum position, FWHM, and the intensity of one-phonon $\mathrm{Si}$ line were taken as equal to the following parameters of fitted Lorentzian function: center of the profile, its FWHM, and the height.

In the case of ultraviolet Raman spectrum the attention was focused on the scattering generated in silicon dioxide layer. In such a case one-phonon Si line disturbs the signal coming from $\mathrm{SiO}_{2}$. This line was reconstructed with fitting Lorentzian profile. Fitted function was subtracted from the measured deep-UV Raman spectra in order to remove onephonon Si line. This procedure of removal of one-phonon Si line was already discussed in the literature [20]. Obtained from subtraction spectrum was assigned to scattering from silicon dioxide layer. Broad bands are generated in the part of the layer which has an amorphous structure. This assignment was discussed in detail previously [20]. In this work narrow lines which appear on the background generated in amorphous form of $\mathrm{SiO}_{2}$ were compared with data reported for different crystalline forms of silicon dioxide.

\section{Results}

Figure 2 presents the analysis of one-phonon Si line obtained from line scan $z$. Maximum position of the line is presented

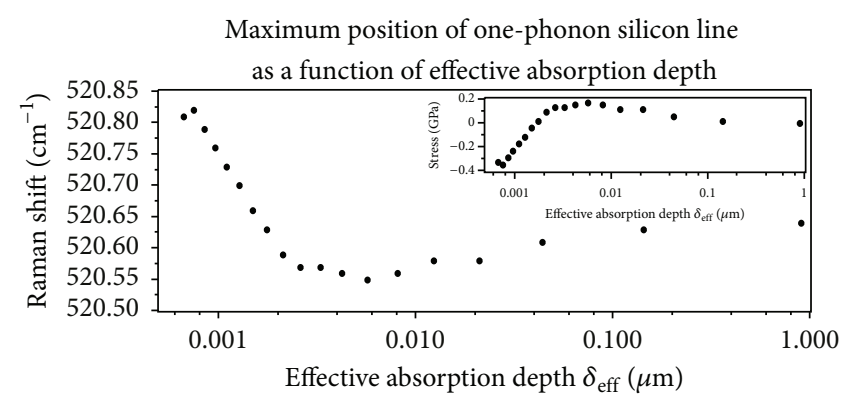

(a)

FWHM of one-phonon silicon line as a function of effective absorption depth

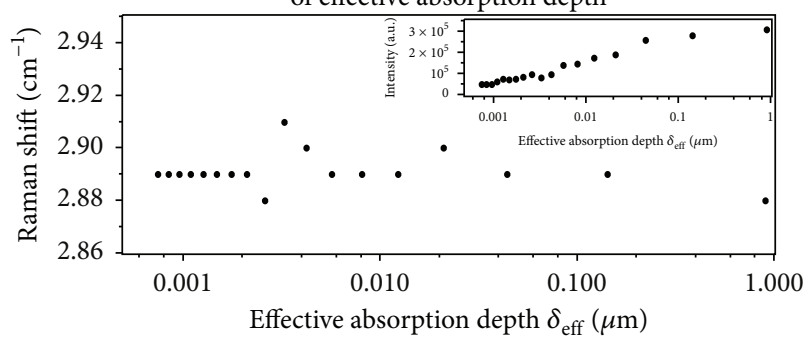

(b)

FIGURE 2: Raman data obtained with excitation in visible spectral range: (a) position of maximum of one-phonon $\mathrm{Si}$ line as a function of effective absorption depth and (b) FWHM of one-phonon Si line as a function of effective absorption depth. Insets present following functions of effective absorption depth: panel (a) mechanical stress in silicon calculated from position of one-phonon Si line and panel (b) intensity of the one-phonon Si line.

in part (a) and the analysis of FWHM of the line is shown in part (b) of the figure.

The main plot in panel (a) shows the maximum position of one-phonon Si line as a function of effective absorption depth. The position of the maximum changes with $\delta_{\text {eff }}$ in the following way:

(i) for values of $\delta_{\text {eff }}$ below $4 \mathrm{~nm}$ the linear decrease of maximum position with the increase of $\delta_{\text {eff }}$ is observed, the range of Raman shift where the maximum position is changed spreads from $520.83 \mathrm{~cm}^{-1}$ to $520.57 \mathrm{~cm}^{-1}$, and the exception is the first point which does not fit to the behavior presented by other points;

(ii) for $\delta_{\text {eff }}$ from the range between $4 \mathrm{~nm}$ and $90 \mathrm{~nm}$ the maximum position reaches the plateau, and the values of the parameter are placed between $520.55 \mathrm{~cm}^{-1}$ and $520.58 \mathrm{~cm}^{-1}$;

(iii) for $\delta_{\text {eff }}$ larger than $90 \mathrm{~nm}$ maximum position increases asymptotically to the limit equal to $520.65 \mathrm{~cm}^{-1}$.

The inset in panel (a) shows the stress calculated from the simple model for uniaxial stress $[4,30]$.

The main panel in part (b) of Figure 2 shows FWHM as a function of effective absorption depth. This dependence may be treated as constant in the whole range of $\delta_{\text {eff. }}$. The values of FWHM are placed within the range $(2.89 \pm 0.02)$ 


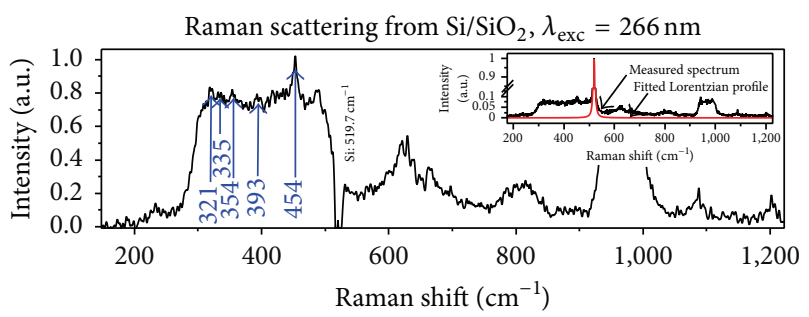

FIGURE 3: Raman spectrum of silicon dioxide layer placed on silicon substrate after removal of one-phonon Si line. Maxima of narrow lines assigned to crystalline forms of silicon dioxide are given in the main plot (blue numbers). The maximum of one-phonon $\mathrm{Si}$ line is also given in main plot (black number). The inset presets the spectrum before removal of one-phonon Si line (black) and Lorentzian function used for reconstruction of silicon line (red).

$\mathrm{cm}^{-1}$. The inset in Figure 2(b) presents the intensity as a function of effective absorption depth. For $\delta_{\text {eff }}$ smaller than $100 \mathrm{~nm}$ intensity increases with the value of $\delta_{\text {eff. }}$. For larger penetration depths than $100 \mathrm{~nm}$ stabilization of the intensity is observed.

The deep-UV Raman spectrum of $\mathrm{SiO}_{2}$ layer is presented in Figure 3. In the main plot one-phonon Si line was removed. Fitted to one-phonon Si line Lorentzian profile was subtracted from the spectrum (as described in previous chapter). The maxima of narrow lines which are compared with data reported for crystalline forms of silicon dioxide are given in the figure. The inset in Figure 3 shows the spectrum before the removal of Si one-phonon line together with Lorentzian function used for reconstruction of one-phonon Si line. In order to present the spectrum coming from $\mathrm{SiO}_{2}$ layer the break in ordinate axis was introduced in the inset.

\section{Discussion}

Before discussion of the spectroscopic data obtained in this work we will sum up the information about $\mathrm{Si} / \mathrm{SiO}_{2}$ interface and its close vicinity. The data from the literature described here will be used to analyze the results of Raman study reported in this work.

The transition area between crystalline $\mathrm{Si}(c-\mathrm{Si})$ and amorphous $\mathrm{SiO}_{2}\left(a-\mathrm{SiO}_{2}\right)$ was the subject of interest already in the seventies of last century [31]. The most simple model of the interface assumed step-like transition between $c$-Si and $a-\mathrm{SiO}_{2}$. Properties of this type of interface were the subject of $a b$ initio theoretical study [32]. The structure of $a$ $\mathrm{SiO}_{2}$ was simulated by means of $\beta$-cristobalite structure [32]. $\beta$-Cristobalite is one of the crystalline forms of $\mathrm{SiO}_{2}$. The results obtained from photoemission spectroscopy suggested gradual type of transition between $a-\mathrm{SiO}_{2}$ and $c$-Si [31]. The transition nonstoichiometric layer was marked as $\mathrm{SiO}_{x}$. The thickness of this layer was estimated as equal to $4 \AA$.

Proposed types of $\mathrm{Si} / \mathrm{SiO}_{2}$ interfaces were divided into three categories due to the intermediate structure between $a$ $\mathrm{SiO}_{2}$ and $c$-Si [33]:

(i) step-like interface means no intermediate layer between $a-\mathrm{SiO}_{2}$ and $c$-Si; (ii) with intermediate $\mathrm{SiO}_{2}$ layer, the intermediate layer is an ordered structure in which crystallographic parameters can match the parameters of $c-\mathrm{Si}$;

(iii) with intermediate $\mathrm{SiO}_{x}$ layer, in this case the intermediate layer is a mixture of silicon and silicon dioxide.

Authors of [33] analyzed following issues related to structure of transition area between $c$-Si and $a-\mathrm{SiO}_{2}$ :

(i) the data obtained with High-Resolution Transmission Electron Microscopy (HRTEM);

(ii) the results of theoretical calculations;

(iii) compliance of crystalline structure of different forms of silicon dioxide and $c$-Si.

They came to following conclusions:

(i) the transition between $a$-SiO $\mathrm{S}_{2}$ and $c$-Si should have the gradual character;

(ii) the thickness of the transition layer should be equal to $5 \AA$ and its structure should be similar to tridymite;

(iii) cristobalite as structure of transition layer should be excluded due to large mismatch of crystalline structure parameters of cristobalite and $c$-Si; the differences reach even $40 \%$ of parameter values.

The structure of the transition area between $a-\mathrm{SiO}_{2}$ and $c$-Si was also investigated by means of spectroscopic ellipsometry [34]. Authors of [34] compared experimental data covering VIS and UV spectral range with different models of the system $a-\mathrm{SiO}_{2} /$ interface/c-Si. They came to the following conclusion: the interface should have gradual character and its thickness should be equal to $7 \AA$. The model assuming step-like transition between $a-\mathrm{SiO}_{2}$ and $c$-Si is a good starting point for investigation of interface structure [35]. However, the statistics coming out from experimental data point to gradual type of interface. It means that intermediate layer should have finite and nonzero thickness [35]. In other words, experimental data suggest gradual character of the transition between $a-\mathrm{SiO}_{2}$ and $c$-Si.

Application of Core-Level Photoemission Spectroscopy (CLPS) confirmed the presence of Si atoms in intermediateoxidation state in the vicinity of interface [36]. The presence of atoms corresponding to following oxidation states $\mathrm{Si}^{1+}$, $\mathrm{Si}^{2+}$, and $\mathrm{Si}^{3+}$ points to gradual character of the interface. In particular atoms $\mathrm{Si}^{3+}$ should not be observed in the case of step-like transition between $a-\mathrm{SiO}_{2}$ and $c$-Si [36]. Authors of [36] proposed that transition area (interface) is composed of two sublayers. One sublayer should be placed in silicon substrate. In this part Si atoms in intermediate-oxidation state should be present. The other component of interface should extend up to $30 \AA$ into the silicon dioxide layer. This area of $\mathrm{SiO}_{2}$ should contain the material which has higher density than the $a-\mathrm{SiO}_{2}$. Also the energy factor related to the stress appearing due to interaction between $a-\mathrm{SiO}_{2}$ layer and $c-\mathrm{Si}$ substrate suggests gradual type of interface [36]. Step-like transition should create larger stress than the transition area having finite and nonzero thickness. The interface model which includes Si atoms in intermediate-oxidation state is 
more favorable than step-like transition due to energetic conditions on interface [37]. Energy related to mismatch of crystalline structures of $a-\mathrm{SiO}_{2}$ and $c$-Si is significantly smaller in the case of models assuming gradual transition between crystalline semiconductor substrate and amorphous dielectric layer [37].

Simulation of the images obtained from HRTEM gives results consistent with experimental data if the gradual type $a-\mathrm{SiO}_{2} / c$-Si interface is assumed [38]. The surface of $\langle 100\rangle$ oriented $c-\mathrm{Si}$ is not smooth if the interface has finite and nonzero thickness $[37,38]$. If smooth $c$-Si surface is assumed the simulated interface becomes step-like. The attempt to simulate more simple interface which takes into account the data obtained from deep electron shells results in the structure called sawhorse [39]. Sawhorse consists of two Si and six $\mathrm{O}$ atoms. $\mathrm{Si}-\mathrm{Si}$ bond is placed parallel to the interface plane. Each $\mathrm{Si}$ atom is bonded to three $\mathrm{O}$ atoms. The sawhorse is bounded to $c$-Si and $a-\mathrm{SiO}_{2}$ via $\mathrm{O}$ atoms. To sum up, the attempt to simplify the interface leads to at least one layer of sawhorses. However, according to discussion in [39] it proposed that model does not take into account defects. Introducing the defects into the model will complicate it and should increase the thickness of the intermediate layer.

Investigation of morphology of $c$ - $\mathrm{Si}\langle 100\rangle / a-\mathrm{SiO}_{2}$ interface by means of Scanning Tunneling Microscopy (STM) showed strong disorder in Si structure in the vicinity of interface. This disordered Si built the layer which is parallel to the interface. The thickness of this layer is of the order of $100 \AA$ [40]. Ion bombardment was used to disclose the interface. The disclosed surface of $c$-Si is not smooth. The hillocks were found on disclosed silicon surface. Horizontal dimension of observed hillocks is equal to about $50 \AA$. The typical height of hillocks is equal to about $10 \AA$ [40].

Ellipsometry investigation of $\langle 100\rangle$ oriented Si wafers after oxidation at different condition showed that the thickness of transition area between silicon substrate and oxide layer depends on the condition of oxidation process [41]. As a model of the transition layer the mixture of amorphous silicon $(a-\mathrm{Si})$ with $\mathrm{SiO}_{2}$ was assumed. The thickness of this interface was of the order of $1 \mathrm{~nm}$. The other result reported in [41] is related to contribution of the signal coming from interface to the whole ellipsometry signal. This contribution decreases with the increase of the thickness of $\mathrm{SiO}_{2}$ layer. This observation led authors of [41] to the conclusion that $\mathrm{SiO}_{2}$ layer can be treated as structure composed of two sublayers. One sublayer with high refractive index is placed in the vicinity of the substrate. This sublayer can be treated as this part of interface (transition area) which is extended into $\mathrm{SiO}_{2}$ layer [36]. The second sublayer is composed of $a-\mathrm{SiO}_{2}$ and can be treated as quasibulk material. Authors of reference [41] came to the conclusion that the interface has certain roughness. This roughness does not depend on the type of $c$-Si surface which is oxidized.

Results obtained by means of Deep Level Transient Spectroscopy (DTLS) suggest the similarity between $a-\mathrm{SiO}_{2} / c-\mathrm{Si}$ interface and amorphous silicon doped with hydrogen ( $a-$ Si:H) [42].

To sum up, transition area between crystalline silicon substrate and amorphous silicon dioxide layer has gradual character. This layer, in the literature marked with $\mathrm{SiO}_{x}$, can be treated as a mixture of amorphous or polycrystalline silicon with silicon dioxide. This transition layer can be divided into two sublayers. The sublayer placed in the vicinity of $c$-Si wafer is characterized by the dominance of silicon structures. The other sublayer is composed of silicon dioxide; however, the density of the dioxide in this sublayer should be larger in comparison with $a-\mathrm{SiO}_{2}$. The optimization of the energy in transition region between $c$ - $\mathrm{Si}$ and $a-\mathrm{SiO}_{2}$ is the reason for the presence of $\mathrm{SiO}_{x}$ layer and its segregation into two sublayers: one with predominance of silicon and the other with oxide structures. Similar segregation of $\mathrm{SiO}_{x}$ layer into two sublayers is also observed for homogeneous $\mathrm{SiO}_{x}$ layer after Rapid Thermal Annealing [43]. One sublayer is dominated by higher concentration of Si structures; in the other sublayer the main component is $\mathrm{SiO}_{2}$.

To discuss obtained data in this work in terms of interface (transition area) that consists of two sublayers analysis two types of Raman signal must be taken into account. The first problem to be discussed is the dependence between shift of one-phonon Si line position and structural changes of the substrate. The other problem to analyze is the presence of the trace of crystalline $\mathrm{SiO}_{2}$ in Raman scattering generated in dioxide layer. Before coming to the obtained results in this work let us first sum up the literature data necessary to assign measured Raman spectra to different form of silicon and silicon dioxide. The changes of the $\mathrm{Si}$ and $\mathrm{SiO}_{2}$ related to different values of absorption depth will be correlated with the literature data concerning transition between $c$-Si and $a$ $\mathrm{SiO}_{2}$ reported above.

At first the dependence between one-phonon line and silicon structure will be reported.

Transition from monocrystalline Si to polycrystalline silicon is observed in Raman spectrum as a shift of onephonon line towards smaller values of Raman shift [44]. The shape of the line becomes asymmetric [44]. The limit of these structural changes is defined by $a$-Si. In the case of $a$-Si the maximum position of one-phonon line is equal to about $480 \mathrm{~cm}^{-1}$. FWHM of this line is many times larger than the FWHM of $c$-Si [45]. Detailed study of the dependence between the type of silicon (crystalline, polycrystalline, or amorphous) and position of one-phonon line was done for $\mathrm{Si}$ layer deposited on different substrates. Presence of all types of material, in particular $c$-Si, polycrystalline $\mathrm{Si}$, and $a$-Si, results in complicated shape of Raman line, because the line is composed of three components: crystalline, polycrystalline, and amorphous [46]. The position of crystalline component can also change in the range of about $2 \mathrm{~cm}^{-1}$. For example, as reported in [46] maximum of Raman line observed for $c$ Si changes from $520.91 \mathrm{~cm}^{-1}$ to $519.17 \mathrm{~cm}^{-1}$ if concentration of defects in crystalline structure increases. Authors of [46] determined the position of one-phonon Raman line for polycrystalline $\mathrm{Si}$ as equal to about $510 \mathrm{~cm}^{-1}$ and, as mentioned above, for $a$-Si as equal to about $480 \mathrm{~cm}^{-1}$.

The other factor that influences the position of maximum of one-phonon line is the admixture gas [46]. Generally, amorphous components are shifted towards smaller values of Raman shift, crystalline to larger. An important parameter 
related to presence of doping gas is its concentration. Small values of hydrogen concentration improve the quality of crystalline structure. Large concentration produces an opposite effect. Authors of [46] also calculated the stress induced by doping agent. They used the formula developed for $a$ Si by Anastassakis. Similar results were obtained for silicon microcapsules containing porous silicon [23]. Authors of [23] assumed that one-phonon Si line is a mixture of two components:

(i) polycrystalline, which has the maximum of onephonon line placed between $515 \mathrm{~cm}^{-1}$ and $520 \mathrm{~cm}^{-1}$;

(ii) amorphous, which has the maximum of one-phonon line placed between $495 \mathrm{~cm}^{-1}$ and $510 \mathrm{~cm}^{-1}$.

The stress in microcapsules was calculated within the approximation of uniaxial stress. As a reference, it means state without stress; the maximum position for monocrystalline $\mathrm{Si}$ was assumed, in particular $520.3 \mathrm{~cm}^{-1}$.

Properties of polycrystalline Si layer depend on its thickness and the deposition temperature. In the case of polycrystalline Si film deposited on monocrystalline Si substrate the structure of the layer depends on the distance from the substrate [47]. If the deposition temperature is equal to $620^{\circ} \mathrm{C}$ maximum position changes gradually with the distance from the substrate or thickness of the layer. In particular, maximum position of one-phonon Si line moves towards smaller values of Raman shift if the thickness of the layer or the distance from monocrystalline Si substrate increases. The dependence of maximum position upon the distance from the monocrystalline substrate is attributed to the changes of crystal dimension. This type of behavior is called phonon confinement effect and can be described as a result of limitation of phonon propagation area caused by small size of crystals [48]. In particular, if average dimension of the $\mathrm{Si}$ crystal decreases from $4.9 \mathrm{~nm}$ to $2.2 \mathrm{~nm}$ the maximum of onephonon line shifts from $519.7 \mathrm{~cm}^{-1}$ to $517.7 \mathrm{~cm}^{-1}$. Described above behavior is not observed if the deposition temperature is from the range between $570^{\circ} \mathrm{C}$ and $590^{\circ} \mathrm{C}$. It suggests that gradual change of crystal dimension with the distance from monocrystalline substrate is caused by temperature. The threshold temperature which activates the process is equal to about $600^{\circ} \mathrm{C}$.

The presence of compressive stress in polycrystalline form is caused by partial oxidation of polycrystalline Si layer [49]. Detailed investigation of partial oxidation of polycrystalline Si layer showed the correlation between the stress and the crystal dimension. The effect is important in the case of small grains [50]. Author of [50] showed the shift of one-phonon Si line corresponding to compressive stress in the case of grains whose diameter is equal to $50 \AA$. If the size of crystalline grains increases to $300 \AA$ the shift of maximum position of one-phonon Si line is not observed. It means that increase of grain dimension over certain limit removes compressive stress caused by local oxidation [50].

Authors of [49] divided silicon into three categories due to maximum position of one-phonon line: crystalline, polycrystalline, and amorphous. Polycrystalline Si layers are characterized by the presence of the stress which cannot be removed even by means of thermal annealing. The value of this nonremovable stress does not exceed $200 \mathrm{MPa}$ [51]. The shift of maximum position of one-phonon silicon line towards smaller values of Raman shift observed for polycrystalline $\mathrm{Si}$ is treated as a trace of tensile stress $[52,53]$. Position of the maximum recorded for $c$-Si was used as a reference. The type of stress in polycrystalline Si layer depends also on the substrate [54]. If glass plate is used as a substrate thermal effects related to cooling of the sample lead to tensile stress [54]. Application of sapphire as a substrate changes the type of stress to compressive [54].

The other point to be discussed is related to the spectrum presented in Figure 3, in particular, to the origin of narrow lines placed on the background composed of Raman scattering assigned to vibrations in $a-\mathrm{SiO}_{2}$ layer [20]. Since these lines are narrow they should be compared with Raman spectra reported for crystalline forms of silicon dioxide. The oxide sublayer of the interface between amorphous silicon dioxide and crystalline silicon [36] should be composed of $\mathrm{SiO}_{2}$ which has the structure similar to crystalline silicon dioxide [21]. Since the crystal-like sublayer is thin observed narrow lines should be compared with strong lines of crystalline forms of $\mathrm{SiO}_{2}$. Let us now complete the information necessary for discussion of the results obtained in this work.

The following strong lines of crystalline forms of silicon dioxide are placed in the same range of Raman shift as the main band of $a-\mathrm{SiO}_{2}$ :

(i) tridymite: $355 \mathrm{~cm}^{-1}, 403 \mathrm{~cm}^{-1}, 422 \mathrm{~cm}^{-1}, 449 \mathrm{~cm}^{-1}$, and $457 \mathrm{~cm}^{-1}$ [55];

(ii) cristobalite: $380 \mathrm{~cm}^{-1}, 426 \mathrm{~cm}^{-1}$ [56], $421 \mathrm{~cm}^{-1}$ [55], and $420 \mathrm{~cm}^{-1}$ [57];

(iii) $\alpha$-quartz: $465 \mathrm{~cm}^{-1}$ [55];

(iv) coesite: $522 \mathrm{~cm}^{-1}$ [55] and $521 \mathrm{~cm}^{-1}$ [58];

(v) moganite: $449 \mathrm{~cm}^{-1}, 463 \mathrm{~cm}^{-1}$, and $501 \mathrm{~cm}^{-1}$ [55].

Due to strong coincidence between one-phonon line generated in $c$-Si and line reported for coesite this crystalline form of $\mathrm{SiO}_{2}$ must be excluded from the discussion of deep-UV Raman scattering.

Let us start the discussion of Raman data obtained in this work from VIS spectral range. The crucial point is the relation between position of one-phonon Si line and the structure of silicon.

Changes of maximum position of one-phonon Si line with the effective absorption depth reflect changes of silicon structure and influence of oxygen which can penetrate into $\mathrm{Si}$ substrate. In the range of effective absorption depth up to $6 \mathrm{~nm}$ polycrystalline or crystalline form with large concentration of defects is expected.

Linear decrease of the maximum position from $520.8 \mathrm{~cm}^{-1}$ to $520.55 \mathrm{~cm}^{-1}$ observed for $\delta_{\text {eff }}$ below $2 \mathrm{~nm}$ should be caused by partial oxidation of Si substrate in this range of effective absorption depth. Oxygen diffused into the $\mathrm{Si}$ substrate due to polycrystalline structure or large concentration of defects. This oxygen caused local oxidation of silicon. The increase of molar volume between $\mathrm{SiO}_{2}$ and $\mathrm{Si}$ results in local stress which has compressive character. 
The amount of oxygen that can penetrate into the substrate decreases with the distance from the interface. This effect is reflected in Raman spectrum as a decrease of maximum position of one-phonon Si line (see Figure 2(a)). The effect observed in this work is similar to local oxidation of polycrystalline silicon reported in [50].

For $\delta_{\text {eff }}$ from the range between $2 \mathrm{~nm}$ and $6 \mathrm{~nm}$ maximum position of one-phonon Si line is stabilized. The value of this plateau is equal to $520.55 \mathrm{~cm}^{-1}$. This position of $\mathrm{Si}$ line suggests that the structure should have crystalline character with significant concentration of defects [49]. Polycrystalline form should be excluded because in such a case the position of one-phonon Si line should be shifted by several $\mathrm{cm}^{-1}$ towards smaller values of Raman shift. In the case of polycrystalline form of silicon the precise position of maximum of onephonon line is a function of grain dimension $[46,48]$.

For $\delta_{\text {eff }}$ larger than $6 \mathrm{~nm}$ the asymptotic increase of maximum position from $520.55 \mathrm{~cm}^{-1}$ to $526.65 \mathrm{~cm}^{-1}$ is observed. This behavior can be interpreted as gradual reduction of tensile stress caused in crystalline form of silicon by interaction with dioxide layer. The asymptotic position $520.65 \mathrm{~cm}^{-1}$ is equal to the mean value of maximum position of one-phonon Si line calculated from data reported in the literature $[4,45$, $50,59,60]$. As reported in the literature values of maximum position of one-phonon line observed for crystalline silicon are placed in the range between $520.3 \mathrm{~cm}^{-1}$ and $521 \mathrm{~cm}^{-1}$.

FWHM observed for different values of $\delta_{\text {eff }}$ is placed in the range between $2.87 \mathrm{~cm}^{-1}$ and $2.91 \mathrm{~cm}^{-1}$. No unequivocal tendency in the dependence between FWHM and $\delta_{\text {eff }}$ can be observed. Because of this FWHM can be treated as constant and equal to $(2.89 \pm 0.02) \mathrm{cm}^{-1}$. Maximum deviation from mean value equal to $0.02 \mathrm{~cm}^{-1}$ is significantly smaller than spectral resolution of the apparatus which is equal to about $0.1 \mathrm{~cm}^{-1}$. The mean value of FWHM equal to $2.89 \mathrm{~cm}^{-1}$ is in agreement with typical values reported for crystalline form of Si $[50,59]$.

To sum up, changes of one-phonon Si line in the vicinity of the border between $a-\mathrm{SiO}_{2}$ and $c$-Si are influenced by three factors:

(i) stress generated due to differences in molar volume between silicon and dioxide arising due to silicon oxidation;

(ii) structure of $\mathrm{Si}$ wafer in the vicinity of the border between substrate and oxide layer;

(iii) effects caused by oxygen diffusion into the thin silicon layer placed in the vicinity of the border between silicon dioxide layer and silicon wafer.

Let us move now to the discussion of the results obtained from deep-UV excitation. As was mentioned before narrow lines placed on the background composed of bands assigned to $a-\mathrm{SiO}_{2}$ should be compared with data reported for crystalline forms of silicon dioxide. Lines $321 \mathrm{~cm}^{-1}$ and $335 \mathrm{~cm}^{-1}$ have no equivalents among strong lines reported for different crystalline forms of $\mathrm{SiO}_{2}$. These lines $\left(321 \mathrm{~cm}^{-1}\right.$ and $\left.335 \mathrm{~cm}^{-1}\right)$ have the best correlation with following weaker lines reported for different crystalline forms of $\mathrm{SiO}_{2}: 330 \mathrm{~cm}^{-1}$ reported for tridymite [55], $328 \mathrm{~cm}^{-1}$ observed for coesite [58], and with $317 \mathrm{~cm}^{-1}$ present in spectra recorded for moganite [55]. Other lines presented in Figure 3 have following correlation with strong lines reported in the literature for different crystalline forms of silicon dioxide:

(i) line with maximum at $354 \mathrm{~cm}^{-1}$ has the best correlation with line centered around $355 \mathrm{~cm}^{-1}$ reported for tridymite;

(ii) line with maximum at $393 \mathrm{~cm}^{-1}$ is placed between lines $380 \mathrm{~cm}^{-1}$ and $403 \mathrm{~cm}^{-1}$ reported for cristobalite and tridymite, respectively;

(iii) line with maximum at $454 \mathrm{~cm}^{-1}$ has best correlation with

(a) lines $449 \mathrm{~cm}^{-1}$ and $457 \mathrm{~cm}^{-1}$ reported for tridymite,

(b) line $449 \mathrm{~cm}^{-1}$ recognized in moganite Raman spectra.

It should be emphasized that the discussed above lines, $354 \mathrm{~cm}^{-1}, 393 \mathrm{~cm}^{-1}$, and $454 \mathrm{~cm}^{-1}$, can be correlated with strong lines reported for tridymite. Tridymite and crystalline silicon show the smallest mismatch between crystalline parameters [33]. This results in favorable energy conditions in the transition area between $a-\mathrm{SiO}_{2}$ and $c$-Si.

Line $454 \mathrm{~cm}^{-1}$ is placed in the same range of Raman shift as bands observed for Si nanocrystals [61]. The conditions of sample preparation in [61] were conductive for creation of nanocrystalline Si grains. In particular thermal annealing of the samples was performed at $1200^{\circ} \mathrm{C}$. The time of this annealing was equal to 2 hours. The surface oxidation of Si substrate is rapid process. The time necessary for manufacturing is below 1 minute. It makes the condition for diffusion of Si atom into $\mathrm{SiO}_{2}$ layer unfavorable, so the line with maximum placed at $454 \mathrm{~cm}^{-1}$ should be assigned rather to $\mathrm{SiO}_{2}$ crystalline form compared to Si nanocrystals.

\section{Summary}

The behavior of Raman spectra shows complex structural changes in the transition area between amorphous silicon dioxide and crystalline silicon. This interface (transition area) has gradual character as was suggested on the basis of experimental techniques like HRTEM, ellipsometry, and CLPS, as well as on the basis of theoretical calculations [3336].

The other important conclusion coming from analysis is the segregation of the interface into two sublayers. The sublayers can be called oxide sublayer and silicon sublayer. Raman spectra registered for visible excitation point to crystalline silicon with large concentration of structural defects or polycrystalline form of silicon as a main component of silicon sublayer of interface. Oxygen diffusion into silicon sublayer is observed as gradual change of one-phonon line maximum position with change of effective absorption depth. Excited in ultraviolet spectral range Raman scattering suggests the presence of oxide sublayer in the area of interface. 
The structure of silicon dioxide placed within this sublayer should be similar to tridymite. This is in agreement with the analysis of the mismatch between the crystalline structure of silicon and different forms of crystalline silicon dioxide [33].

This work presents also the methodology of the investigation that makes possible the extraction of the information from thin layer near the border between silicon dioxide and silicon. The key property that makes this extraction possible is the absorption of the exciting radiation by material under study. By variation of power density of the exciting light the effective absorption depth may be changed. In this way the signal from different parts of the studied sample can be recorded. An important point of the investigation is the development of the model that makes link measured Raman scattering possible with the effective absorption depth. As a result the analysis of material properties as a function of absorption depth is possible.

\section{Conflict of Interests}

The author declares that there is no conflict of interests regarding the publication of this paper.

\section{References}

[1] R. Lai, X. B. Mei, W. R. Deal et al., "Sub 50 nm InP HEMT device with Fmax greater than $1 \mathrm{THz}$, in Proceedings of the IEEE International Electron Devices Meeting (IEDM '07), pp. 609-611, IEEE, Washington, DC, USA, December 2007.

[2] K. Krol, M. Sochacki, M. Turek et al., "Influence of nitrogen implantation on electrical properties of $\mathrm{Al} / \mathrm{SiO}_{2} / 4 \mathrm{H}-\mathrm{SiC} \mathrm{MOS}$ structure," Materials Science Forum, vol. 740-742, pp. 733-736, 2013.

[3] A. Sarua, J. Hangfeng, M. Kuball et al., "Integrated microRaman/infrared thermography probe for monitoring of selfheating in AlGaN/GaN transistor structures," IEEE Transactions on Electron Devices, vol. 53, no. 10, pp. 2438-2447, 2006.

[4] I. de Wolf, "Micro-Raman spectroscopy to study local mechanical stress in silicon integrated circuits," Semiconductor Science and Technology, vol. 11, no. 2, pp. 139-154, 1996.

[5] S. J. Harris, A. E. O'Neill, W. Yang et al., "Measurement of the state of stress in silicon with micro-Raman spectroscopy," Journal of Applied Physics, vol. 96, no. 12, pp. 7195-7201, 2004.

[6] L.-Y. Yang, X.-Y. Xue, K. Zhang, X.-F. Zheng, X.-H. Ma, and Y. Hao, "Channel temperature determination of a multifinger $\mathrm{AlGaN} / \mathrm{GaN}$ high electron mobility transistor using a microRaman technique," Chinese Physics B, vol. 21, no. 7, Article ID 077304, 2012.

[7] G. Zhang, S. Feng, J. Li, Y. Zhao, and C. Guo, "Determination of channel temperature for AlGaN/GaN HEMTs by high spectral resolution micro-Raman spectroscopy," Journal of Semiconductors, vol. 33, no. 4, Article ID 044003, 2012.

[8] C. J. de Grauw, N. M. Sijtsema, C. Otto, and J. Greve, "Axial resolution of confocal raman microscopes: gaussian beam theory and practice," Journal of Microscopy, vol. 188, no. 3, pp. 273-279, 1997.

[9] P. Borowicz, A. Kuchuk, Z. Adamus et al., "Visible and deepultraviolet Raman spectroscopy as a tool for investigation of structural changes and redistribution of carbon in ni-based ohmic contacts on silicon carbide," ISRN Nanomaterials, vol. 2012, Article ID 852405, 11 pages, 2012.

[10] K. C. Chang, N. T. Nuhfer, L. M. Porter, and Q. Wahab, "High-carbon concentrations at the silicon dioxide-silicon carbide interface identified by electron energy loss spectroscopy," Applied Physics Letters, vol. 77, no. 14, pp. 2186-2188, 2000.

[11] W. Lu, L. C. Feldman, Y. Song et al., "Graphitic features on SiC surface following oxidation and etching using surface enhanced Raman spectroscopy," Applied Physics Letters, vol. 85, no. 16, pp. 3495-3497, 2004.

[12] Y. Sasaki, Y. Nishina, M. Sato, and K. Okamura, "Raman study of SiC fibres made from polycarbosilane," Journal of Materials Science, vol. 22, no. 2, pp. 443-448, 1987.

[13] A. Gavrikov, A. Knizhnik, A. Safonov et al., "First-principlesbased investigation of kinetic mechanism of SiC(0001) dry oxidation including defect generation and passivation," Journal of Applied Physics, vol. 104, no. 9, Article ID 093508, 2008.

[14] A. C. Ferrari and J. Robertson, "Interpretation of Raman spectra of disordered and amorphous carbon," Physical Review BCondensed Matter and Materials Physics, vol. 61, no. 20, Article ID 14095, 2000.

[15] J. C. Burton, L. Sun, F. H. Long, Z. C. Feng, and I. T. Ferguson, "First- and second-order Raman scattering from semi-insulating 4H-SiC," Physical Review B, vol. 59, no. 11, pp. 7282-7284, 1999.

[16] W. Windl, K. Karch, P. Pavone et al., "Second-order Raman spectra of SiC: experimental and theoretical results from $a b$ initio phonon calculations," Physical Review B, vol. 49, no. 13, pp. 8764-8767, 1994.

[17] P. Borowicz, T. Gutt, T. Małachowski, and M. Latek, "Carbonic inclusions on $\mathrm{SiC} / \mathrm{SiO}_{2}$ interface investigated with Raman Scattering," Diamond \& Related Materials, vol. 20, no. 5-6, pp. 665-674, 2011.

[18] P. A. Temple and C. E. Hathaway, "Multiphonon Raman spectrum of silicon," Physical Review B, vol. 7, no. 8, pp. 36853697, 1973.

[19] A. Chabli, "Optical characterization of layers for silicon microelectronics," Microelectronic Engineering, vol. 40, no. 3-4, pp. 263-274, 1998.

[20] P. Borowicz, M. Latek, W. Rzodkiewicz, A. Łaszcz, A. Czerwinski, and J. Ratajczak, "Deep-ultraviolet Raman investigation of silicon oxide: thin film on silicon substrate versus bulk material," Advances in Natural Sciences: Nanoscience \& Nanotechnology, vol. 3, no. 4, Article ID 045003, 2012.

[21] A. G. Revesz and H. L. Hughes, "The structural aspects of non-crystalline $\mathrm{SiO}_{2}$ films on silicon: a review," Journal of NonCrystalline Solids, vol. 328, no. 1-3, pp. 48-63, 2003.

[22] V. A. Volodin and V. A. Sachkov, "Improved model of optical phonon confinement in silicon nanocrystals," Journal of Experimental and Theoretical Physics, vol. 116, no. 1, pp. 87-94, 2013.

[23] D. Naumenko, V. Snitka, M. Duch, N. Torras, and J. Esteve, "Stress mapping on the porous silicon microcapsules by Raman microscopy," Microelectronic Engineering, vol. 98, pp. 488-491, 2012.

[24] Y. Ohno, M. Akita, S. Kishimoto, K. Maezawa, and T. Mizutani, "Temperature distributions in AlGaN/GaN HEMTs measured by micro-Raman scattering spectroscopy," Physica Status Solidi (C), no. 1, pp. 57-60, 2002.

[25] S. Rajasingam, J. W. Pomeroy, M. Kuball et al., "Micro-Raman temperature measurements for electric field assessment in active AlGaN-GaN HFETs," IEEE Electron Device Letters, vol. 25, no. 7, pp. 456-458, 2004. 
[26] J. Jasny, "Multifunctional spectrofluorimetric system," Journal of Luminescence, vol. 17, no. 2, pp. 149-173, 1978.

[27] N. V. Tkachenko, "Introduction," in Optical Spectroscopy. Methods and Instrumentation, chapter 1, pp. 2-5, Elsevier, 2006.

[28] M. Born and E. Wolf, "Optics of metals," in Principles of Optics: Electromagnetic Theory of Propagation, Interference and Diffraction of Light, chapter 13, p. 614, Pergamon Press, 1970.

[29] M. N. Polyanskiy, "Refractive index database," 2015, http:// refractiveindex.info/.

[30] B. Dietrich, V. Bukalo, A. Fischer et al., "Raman-spectroscopic determination of inhomogeneous stress in submicron silicon devices," Applied Physics Letters, vol. 82, no. 8, pp. 1176-1178, 2003.

[31] T. H. DiStefano, "Field dependent internal photoemission probe of the electronic structure of the $\mathrm{Si}-\mathrm{SiO}_{2}$ interface," Journal of Vacuum Science \& Technology, vol. 13, no. 4, pp. 856-859, 1976.

[32] F. Herman and R. V. Kasowski, "Electronic structure of defects at $\mathrm{Si} / \mathrm{SiO}_{2}$ interfaces," Journal of Vacuum Science \& Technology, vol. 19, no. 3, pp. 395-401, 1981.

[33] A. Ourmazd, D. W. Taylor, J. A. Rentschler, and J. Bevk, "Si $\rightarrow$ $\mathrm{SiO}_{2}$ transformation: interfacial structure and mechanism," Physical Review Letters, vol. 59, no. 2, pp. 213-216, 1987.

[34] D. E. Aspnes and J. B. Theeten, "Optical properties of the interface between Si and its thermally grown oxide," Physical Review Letters, vol. 43, no. 14, pp. 1046-1050, 1979.

[35] C. R. Helms, "Morphology and electronic structure of Si$\mathrm{SiO}_{2}$ interfaces and Si surfaces," Journal of Vacuum Science \& Technology, vol. 16, no. 2, pp. 608-614, 1979.

[36] F. J. Himpsel, F. R. McFeely, A. Taleb-Ibrahimi, J. A. Yarmoff, and G. Hollinger, "Microscopic structure of the $\mathrm{SiO}_{2} / \mathrm{Si}$ interface," Physical Review B, vol. 38, no. 9, pp. 6084-6096, 1988.

[37] I. Ohdomari, H. Akatsu, Y. Yamakoshi, and K. Kishimoto, "Study of the interfacial structure between Si (100) and thermally grown $\mathrm{SiO}_{2}$ using a ball-and-spoke model," Journal of Applied Physics, vol. 62, no. 9, pp. 3751-3754, 1987.

[38] I. Ohdomari, T. Mihara, and K. Kai, "Computer simulation of high-resolution transmission electron microscope images based on ball-and-spoke models of (100) $\mathrm{Si} / \mathrm{SiO}_{2}$ interface," Journal of Applied Physics, vol. 60, no. 11, pp. 3900-3904, 1986.

[39] M. M. Banaszak Holl, S. Lee, and F. R. McFeely, "Core level photoemission and the structure of the $\mathrm{Si} / \mathrm{SiO}_{2}$ interface: a reappraisal," Applied Physics Letters, vol. 65, no. 9, pp. 1097-1099, 1994.

[40] R. M. Feenstra and G. S. Oehrlein, "Surface morphology of oxidized and ion-etched silicon by scanning tunneling microscopy," Applied Physics Letters, vol. 47, no. 2, pp. 97-99, 1985.

[41] C. Zhao, P. R. Lefebvre, and E. A. Irene, "A spectroscopic immersion ellipsometry study of $\mathrm{SiO}_{2}$-Si interface roughness for electron cyclotron resonance plasma and thermally oxidized Si surfaces," Thin Solid Films, vol. 313-314, pp. 286-291, 1998.

[42] N. M. Johnson, D. K. Biegelsen, M. D. Moyer, S. T. Chang, E. H. Poindexter, and P. J. Caplan, "Characteristic electronic defects at the $\mathrm{Si}-\mathrm{SiO}_{2}$ interface," Applied Physics Letters, vol. 43, no. 6, pp. 563-565, 1983.

[43] B. J. Hinds, F. Wang, D. M. Wolfe, C. L. Hinkle, and G. Lucovsky, "Investigation of postoxidation thermal treatments of $\mathrm{Si} / \mathrm{SiO}_{2}$ interface in relationship to the kinetics of amorphous Si suboxide decomposition," Journal of Vacuum Science and Technology B, vol. 16, no. 4, pp. 2171-2176, 1998.
[44] M. D. Efremov, V. V. Bolotov, V. A. Volodin, and S. A Kochubei, "Raman scattering anisotropy in a system of $\left(\begin{array}{lll}1 & 1 & 0\end{array}\right)$ oriented silicon nanocrystals formed in a-Si film," Solid State Communications, vol. 108, no. 9, pp. 645-648, 1998.

[45] I. de Wolf, C. Jian, and W. M. van Spengen, "The investigation of microsystems using Raman spectroscopy," Optics and Lasers in Engineering, vol. 36, no. 2, pp. 213-223, 2001.

[46] Z. P. Ling, J. Ge, R. Stangl, A. G. Aberle, and T. Mueller, "Detailed micro raman spectroscopy analysis of doped silicon thin film layers and its feasibility for heterojunction silicon wafer solar cells," Journal of Materials Science and Chemical Engineering, vol. 1, no. 5, Article ID 38124, pp. 1-14, 2013.

[47] A. A. Parr, C. Bodart, D. Demonchy, and D. J. Gardiner, "Depth profiling variously deposited LPCVD polysilicon films using Raman microscopy," Semiconductor Science and Technology, vol. 16, no. 7, pp. 608-613, 2001.

[48] K. W. Adu, Q. Xiong, H. R. Gutierrez, G. Chen, and P. C. Eklund, "Raman scattering as a probe of phonon confinement and surface optical modes in semiconducting nanowires," Applied Physics A, vol. 85, no. 3, pp. 287-297, 2006.

[49] A. A. Parr, D. J. Gardiner, R. T. Carline, D. O. King, and G. M. Williams, "Structural variations in polysilicon, associated with deposition temperature and degree of anneal," Journal of Materials Science, vol. 36, no. 1, pp. 207-212, 2001.

[50] M. Kawata, S. Nadahara, J. Shiozawa, M. Watanabe, and T. Katoda, "Characterization of stress in doped and undoped polycrystalline silicon before and after annealing or oxidation with laser raman spectroscopy," Journal of Electronic Materials, vol. 19, no. 5, pp. 407-411, 1990.

[51] R. C. Teixeira, I. Doi, M. B. P. Zakia, J. A. Diniz, and J. W. Swart, "Micro-raman stress characterization of polycrystalline silicon films grown at high temperature," Materials Science and Engineering B, vol. 112, no. 2-3, pp. 160-164, 2004.

[52] X.-Z. Bo, N. Yao, S. R. Shieh, T. S. Duffy, and J. C. Sturm, "Largegrain polycrystalline silicon films with low intragranular defect density by low-temperature solid-phase crystallization without underlying oxide," Journal of Applied Physics, vol. 91, no. 5, pp. 2910-2915, 2002.

[53] A. Ogura, K. Egami, and M. Kimura, "Minimization of residual stress in SOI films by using AlN interlaid insulator," Japanese Journal of Applied Physics, vol. 24, no. 8, pp. L669-L671, 1985.

[54] K. Kitahara, T. Ishii, J. Suzuki, T. Bessyo, and N. Watanabe, "Characterization of defects and stress in polycrystalline silicon thin films on glass substrates by raman microscopy," International Journal of Spectroscopy, vol. 2011, Article ID 632139, 14 pages, 2011.

[55] K. J. Kingma and R. J. Hemley, "Raman spectroscopic study of microcrystalline silica," American Mineralogist, vol. 79, no. 3-4, pp. 269-273, 1994.

[56] M. Zhang and J. F. Scott, "Raman studies of oxide minerals: a retrospective on cristobalite phases," Journal of Physics Condensed Matter: Condensed Matter, vol. 19, no. 27, Article ID 275201, 2007.

[57] D. C. Palmer, R. J. Hemley, and C. T. Prewitt, "Raman spectroscopic study of high-pressure phase transitions in cristobalite," Physics \& Chemistry of Minerals, vol. 21, no. 8, pp. 481-488, 1994.

[58] P. Mohanty, V. Ortalan, N. D. Browning, I. Arslan, Y. Fei, and K. Landskron, "Direct formation of mesoporous coesite single crystals from periodic mesoporous silica at extreme pressure," Angewandte Chemie, vol. 49, no. 25, pp. 4301-4305, 2010. 
[59] N. Nakano, L. Marville, and R. Reif, "Raman scattering in polycrystalline silicon doped with boron," Journal of Applied Physics, vol. 72, no. 8, pp. 3641-3647, 1992.

[60] P. Zorabedian and F. Adar, "Measurement of local stress in laserrecrystallized lateral epitaxial silicon films over silicon dioxide using Raman scattering," Applied Physics Letters, vol. 43, no. 2, pp. 177-179, 1983.

[61] V. A. Volodin, M. D. Efremov, V. A. Gritsenko, and S. A. Kochubei, "Raman study of silicon nanocrystals formed in $\mathrm{SiNx}$ films by excimer laser or thermal annealing," Applied Physics Letters, vol. 73, no. 9, pp. 1212-1214, 1998. 

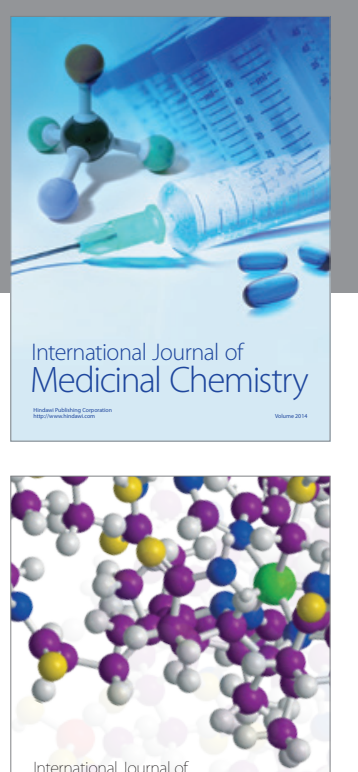

Carbohydrate Chemistry

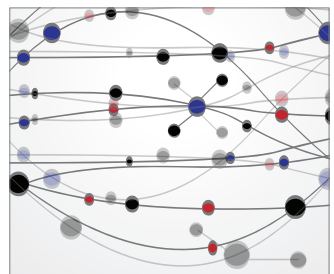

The Scientific World Journal
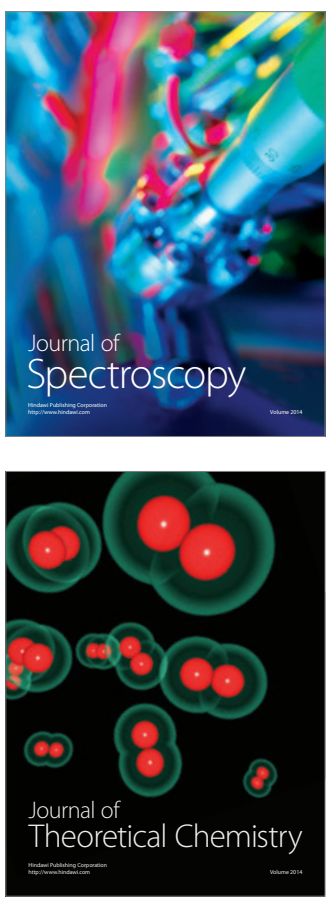
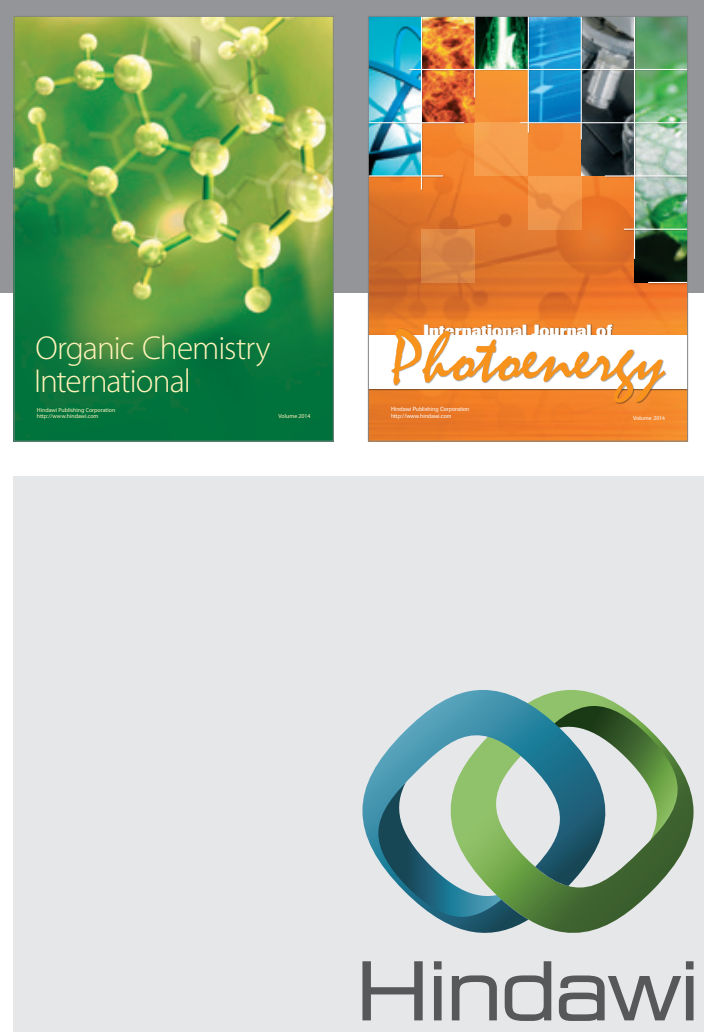

Submit your manuscripts at

http://www.hindawi.com

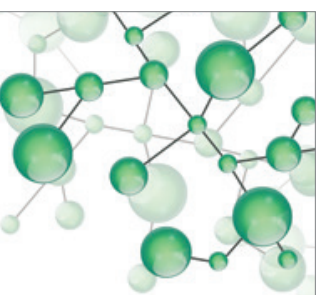

International Journal of

Inorganic Chemistry

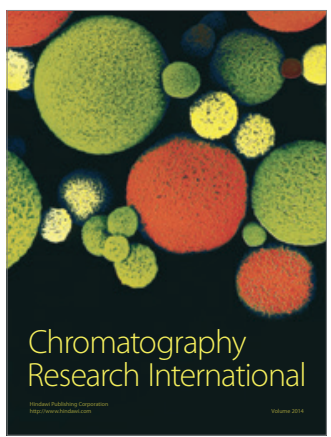

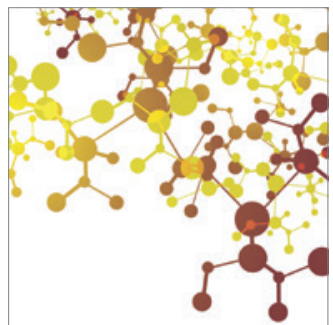

Applied Chemistry
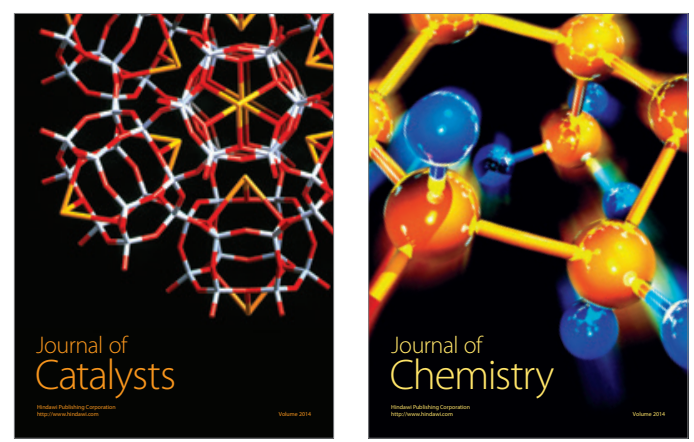
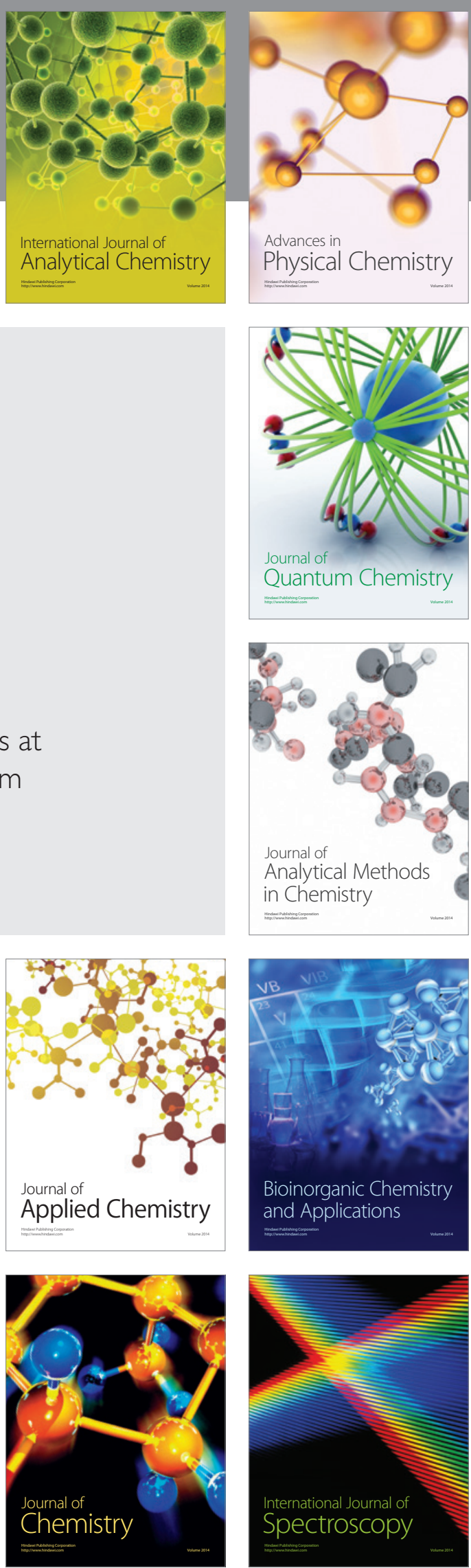